\title{
O ESTADO DA ARTE DO ENSINO DA QUÍMICA NO BRASIL, 2000-2014
}

State of the art of chemistry teaching in Brazil, 2000-2014

\author{
FRANCISCO EDILSON ISAIAS DE SOUSA ${ }^{1}$ \\ DANIEL SILAS VERAS-DOS-SANTOS ${ }^{2}$ \\ EDNA NASCIMENTO OLIVEIRA VERAS ${ }^{3}$
}

RESUMO: Este estudo trata-se de uma revisão bibliográfica do tipo descritiva, comumente denominada "pesquisas do estado da arte", relacionada aos conceitos do Ensino de Química no Brasil. Foi realizada uma busca e levantamento de publicações referentes ao Ensino de Química no Brasil junto aos seguintes bancos de dados: SciELO, Biblioteca Digital da USP, Google Acadêmico e Periódicos da Capes, disponíveis na Internet no período de 2000 a 2014. A busca foi realizada inserindo as palavras-chave: Ensino de Química, Educação em Química e História da Química. Foram analisados 25 artigos, cinco teses e seis dissertações, totalizando 36 publicações sobre a temática o Ensino da Química no Brasil. Foi identificado que o campo da pesquisa em Ensino de Química vem apresentando grande desenvolvimento e interesse cada vez maior por parte da comunidade acadêmica mostrando um amadurecimento dos programas de Pós-Graduação em Ensino de Química. Por outro lado, as pesquisas são ainda sustentadas por uma pequena quantidade de pesquisadores de uma região específica - sudeste. A USP destacouse como a de maior produtividade. A UFPI foi a única Universidade representante do estado do Piauí a produzir trabalho sobre o Ensino de Química. No que se refere aos níveis, a maior parte dos trabalhos tratam-se de aspectos relacionados ao ensino superior. O ensino fundamental necessita-se de maior atenção. O tema mais encontrado nas análises foi recursos didáticos. Espera-se que este trabalho possa colaborar de alguma maneira para a comunidade acadêmica, ainda que de forma preliminar e dado o seu caráter pioneiro na área, contribuir apontando para os conhecimentos produzidos, traçando algumas de suas tendências e disponibilizando materiais para consulta e subsídios para futuras pesquisas.

Palavras-chave: Ensino de Química; Educação em Química; História da Química.

\footnotetext{
${ }^{1}$ Professor da Secretária Estadual de Educação do Piauí. Email: edilsonisaias@,hotmail.com

2 Professor do Instituto Federal do Maranhão Campus Caxias. Email: daniel.veras@ifma.edu.br

${ }^{3}$ Acadêmica de Pedagogia da Faculdade de Tecnologia e Educação Superior Profissional. Email: ednajfmorais@gmail.com
} 
ABSTRACT: This study deals with a literature review descriptive, usually known as "state of the art research" related to the concepts of Chemistry Teaching in Brazil. Search and survey of publications for the Teaching of Chemistry in Brazil with the following databases was conducted: SciELO, USP Digital Library, Google Scholar and Periodicals Capes, available on the Internet from 2000 to 2014. The search was conducted by entering the keywords: Chemistry Teaching, Education in Chemistry and History of Chemistry. Were analyzed 25 articles, six dissertations and theses five, totaling 36 publications on the subject of the chemistry education in Brazil. Was identified that the field of research in Chemistry Teaching has presented great development and growing interest from the academic community showing a ripening of Postgraduate programs in Chemistry Teaching. On the other hand, the research is supported by a small amount of a specific researchers - southeast. USP stands out as the highest productivity. The UFPI was the only representative of the University of Piauí state to produce work on the Chemistry Teaching. Regarding to the levels in most studies dealing with aspects related to higher education. The elementary school needs is more attention. The subject found in most analyzes was didactic resources. It is hoped that this work can contribute in some way to the academic community, albeit in a preliminary form and given its pioneering character in the area, pointing out contribute to the knowledge produced by tracing some of its trends and providing materials for consultation and subsidies future research.

Keywords: Chemistry Teaching; Education in Chemistry; History of Chemistry

\section{INTRODUÇÃO}

Nas últimas décadas tem-se observado uma crescente preocupação na diversificação de metodologias no ensino de química, a utilização de estratégias inovadoras vem ocorrendo tanto no ensino básico quanto no ensino superior (SANTOS Jr. et al., 2016; VALDERRAMA et al., 2016; BRANCO et al., 2015; MIRANDA; SUAR; MARCONDES, 2015).

Assim tem-se observado um consenso construtivista para o ensino de química quando tais abordagens consideram em seu processo de ensino-aprendizagem a história da química, a experimentação e simulação em computadores, o ensino por investigação e a produção de materiais didáticos para ensino no contexto Ciência-Tecnologia-Sociedade-Ambiente (CACHAPUZ et al., 2011; RAMOS; MUÑOZ, 2015; SANTOS Jr. et al., 2016; SILVA; MARCONDES, 2015).

Essas novas propostas visam a aproximar a construção do conhecimento dos alunos mediante uma integração entre os conteúdos específicos e os processos de produção desse 
mesmo conteúdo, principalmente em um contexto de transformação vertiginosa do mundo, que exige respostas adequadas para as demandas contemporâneas, principalmente garantindo o exercício da cidadania plena (CARVALHO, et al., 2012; ROGADO, 2009; NUNES, et al., 2009).

A área de pesquisa em Ensino de Química no Brasil vem se destacando, nas últimas décadas, como um novo campo de pesquisa.

O desenvolvimento de um novo campo de conhecimentos aparece quase sempre associado a condições como: a existência de uma problemática relevante, suscetível de despertar um interesse suficiente que justifique os esforços necessários ao seu estudo; o caráter específico dessa problemática, que impeça o seu estudo por outro corpo de conhecimento já existente e o contexto sociocultural, bem como os recursos humanos e condições externas (CACHAPUZ et al, 2001, p. 157).

Além disso, a pesquisa acadêmica no campo de Ensino de Química em programas de pósgraduação vem se desenvolvendo no Brasil de maneira expressiva havendo uma produção significativa de conhecimentos.

Considerando a produção nesse campo do conhecimento o presente trabalho buscou verificar o "Estado da Arte" do Ensino de Química no Brasil, bem como gerar informações sobre as possíveis lacunas e carências no Ensino de Química.

\section{METODOLOGIA}

A presente pesquisa trata-se de um estudo de revisão bibliográfica do tipo descritiva relacionada ao campo do Ensino de Química no Brasil. Estudos de descrição e análise da produção acadêmica em determinado campo, comumente denominados "pesquisas do estado da arte", permitem conhecer melhor essa produção.

Tal produção merece ser divulgada e estudada de modo amplo e adequada, buscando-se estabelecer as principais tendências da pesquisa neste campo e suas contribuições para a educação no país, bem como mostrar lacunas da produção ainda não cobertas pelos pesquisadores da área (FERREIRA, 2002).

O levantamento de publicações referentes ao Ensino de Química no Brasil foi realizado junto aos seguintes bancos de dados: SciELO, Biblioteca Digital da USP, Google Acadêmico e Periódicos da Capes, disponíveis na Internet. A busca foi realizada inserindo as palavras-chave: Ensino de Química, Educação em Química e História da Química.

Após ser realizado o levantamento, preliminar e descritivo de maneira minuciosa nos bancos virtuais. Realizou-se a leitura cuidadosa dos títulos dos trabalhos e seus respectivos 
resumos, para determinação de sua classificação quanto pertencente ou não ao grupo de trabalhos procurados.

\section{RESULTADOS E DISCUSSÃO}

O levantamento possibilitou a analise 25 artigos da base de dados SciELO e seis dissertações e cinco teses nas seguintes bases de dados: Biblioteca Digital da USP, Google Acadêmico e Periódicos da Capes, totalizando 36 publicações sobre a temática o Ensino da Química no Brasil no período de 2000 a 2014.

$\mathrm{Na}$ figura 1 pode ser observado a relação entre a frequência de publicações relacionadas ao ensino de química por região do país.

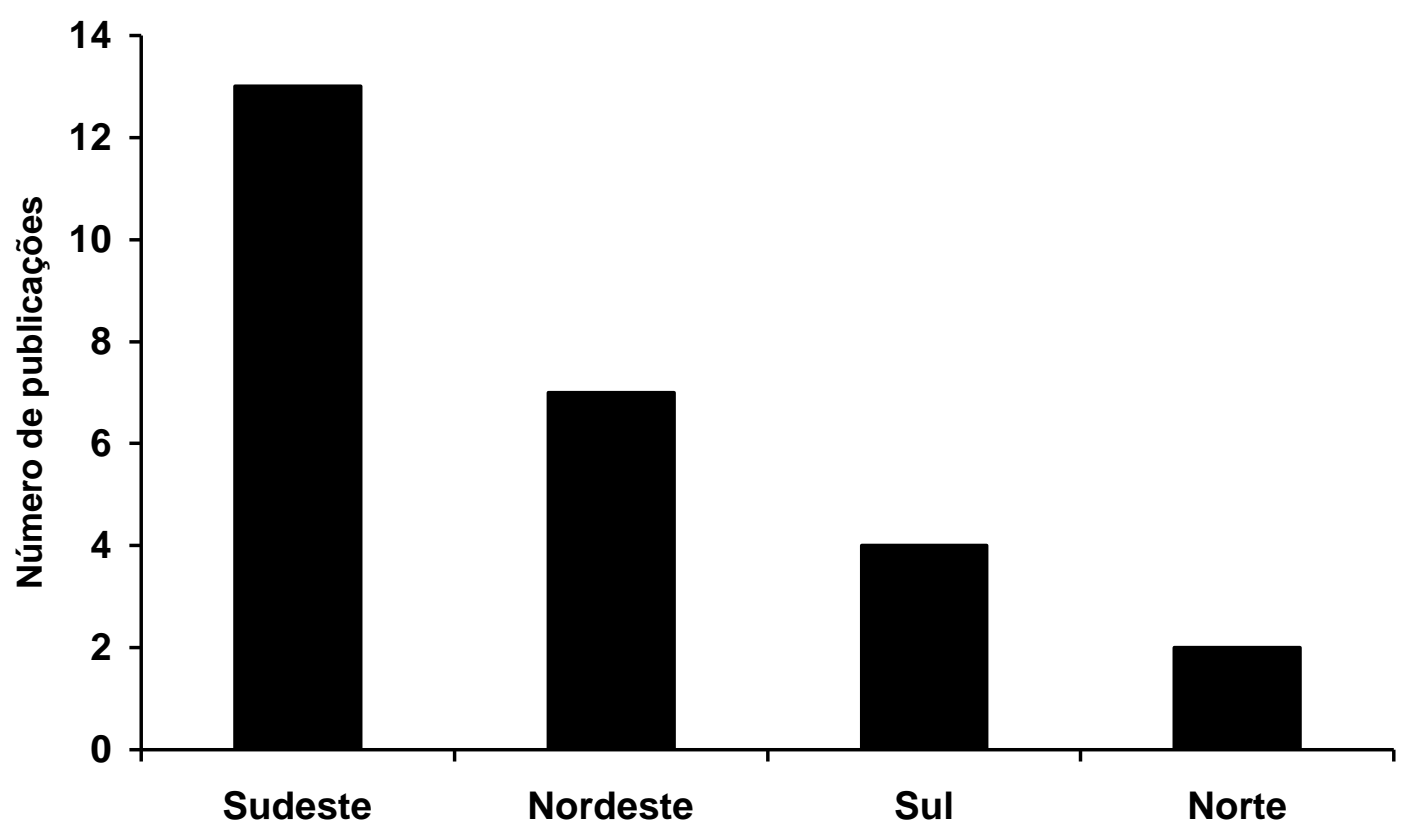

Figura 1: Distribuição do número de publicações sobre o tema Ensino de Química no Brasil em relação à região do país no período de 2000 a 2014.

Os valores observados são similares as produções realizadas de 20041 2008, no qual o Sudeste possuía $53,8 \%$ da produção na área, sendo que a instituição com a maior frequência de trabalhos era a USP com 57\% (FRANCISCO; QUEIROZ, 2010).

Na tabela 1 pode ser acompanhada o número de produções por Instituição de ensino superior, e se percebe que tal situação ainda se mantém.

\section{Instituições}

Porcentagem

Universidade de São Paulo - USP

$20 \%$ 


\begin{tabular}{|c|c|}
\hline Pontifícia Universidade Católica de São Paulo - PUCSP & $4 \%$ \\
\hline Universidade do Estado do Rio de Janeiro - UERJ & $4 \%$ \\
\hline Universidade Federal do Rio de Janeiro - UFRJ & $4 \%$ \\
\hline Universidade Federal da Bahia - UFBA & $8 \%$ \\
\hline Universidade Federal Rural de Pernambuco - UFRPE & $4 \%$ \\
\hline Universidade Federal da Paraíba - UFPB & $4 \%$ \\
\hline Universidade Metodista de Piracicaba - UNIMEP & $4 \%$ \\
\hline Universidade Estadual do Ceará - UECE & $4 \%$ \\
\hline Universidade Federal de Santa Catarina - UFSC & $8 \%$ \\
\hline Universidade Federal do Piauí - UFPI & $4 \%$ \\
\hline $\begin{array}{l}\text { Pontifícia Universidade Católica do Rio Grande do Sul - } \\
\qquad \text { PUCRS }\end{array}$ & $8 \%$ \\
\hline $\begin{array}{l}\text { Universidade Estadual Paulista Júlio de Mesquita Filho - } \\
\text { UNESP }\end{array}$ & $4 \%$ \\
\hline Universidade Estadual de Campinas - UNICAMP & $4 \%$ \\
\hline Universidade Estadual de Santa Cruz - UESC & $4 \%$ \\
\hline Universidade Federal de Minas Gerais - UFMG & $8 \%$ \\
\hline Universidade do Estado do Pará - UEPA & $4 \%$ \\
\hline
\end{tabular}

Tabela 1: Instituições que produziram sobre o Ensino de Química no Brasil e sua porcentagem no período de 2000 a 2014.

O crescimento da produção em outros centros, localizados fora dos programas de pósgraduação das duas universidades paulistas (USP e Unicamp), especialmente na Universidade de Mato Grosso, localizada na região centro-oeste, mostrando uma tendência de descentralização dos centros produtores de pesquisa em Educação Química.

Dos trabalhos analisados verificou-se quanto ao nível de ensino destinados os resultados observados na Figura 2. 


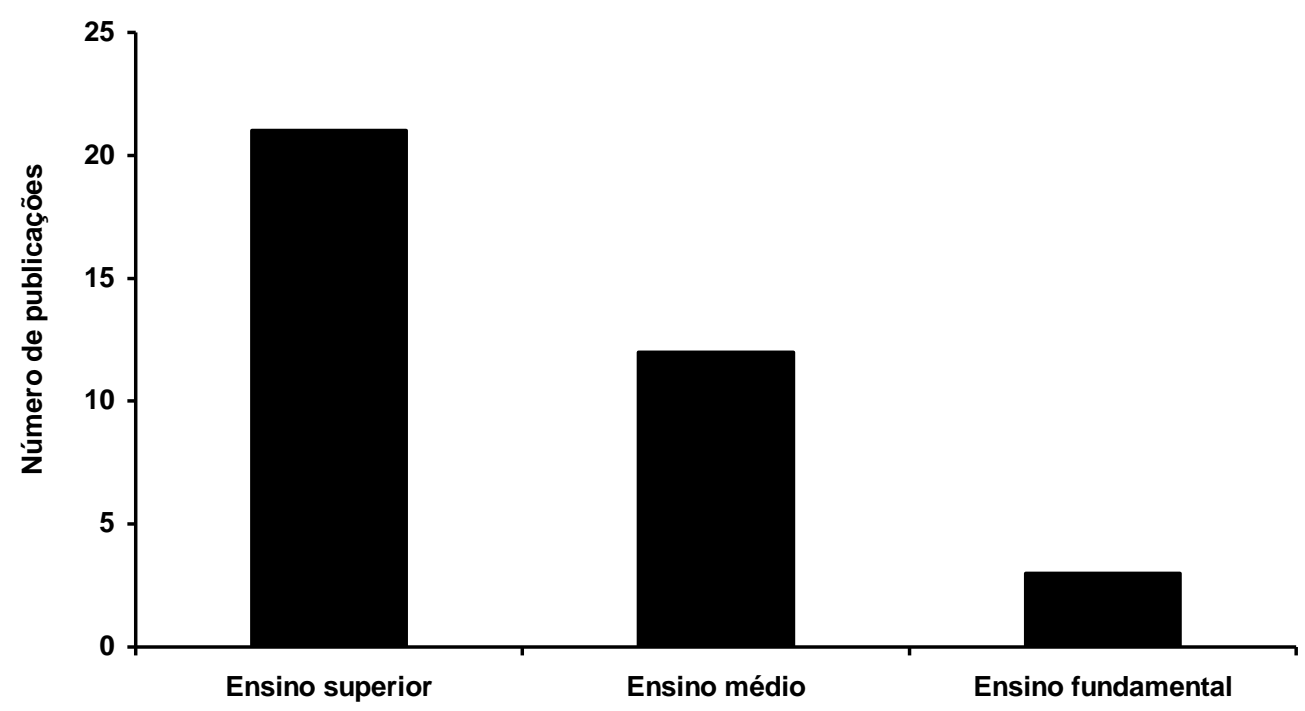

Figura 2: Distribuição do número de publicações sobre o tema Ensino de Química no Brasil em relação ao nível de escolaridade de 2000 a 2014.

O número significativo de trabalhos desenvolvidos no ensino superior pode estar ligado a necessidade de uma formação que supere a grande discordância da natureza da ciência que justificam o fracasso de um bom número de estudantes, e assim permitir uma formação que garanta a compreensão das interações entre ciência, tecnologia, sociedade e ambiente, assim como possibilitar o desenvolvimento da capacidade de resolver problemas e tomar decisões que considerem aspectos sociais, históricos, éticos (CACHAPUZ et al., 2011; SILVA; MARCONDES, 2015).

No ensino básico principalmente no fundamental (11-14 anos), via de regra, conteúdos de Química são vistos no último ano, e apenas no segundo semestre, o que pode explicar a baixa frequência de trabalhos nesse nível de ensino, além de no ensino básico as abordagens serem mais propedêuticas, o que implica muitas vezes em uma impossibilidade na formação para cidadania ou para outro objetivo educacional; ou seja, sua desestruturação é tal que a maioria dos entrevistados afirmou que ele não serve para nada (SANTOS ; SCHETZLER, 1996).

As principais temáticas trabalhadas nas pesquisas podem ser observadas na Figura 3. 


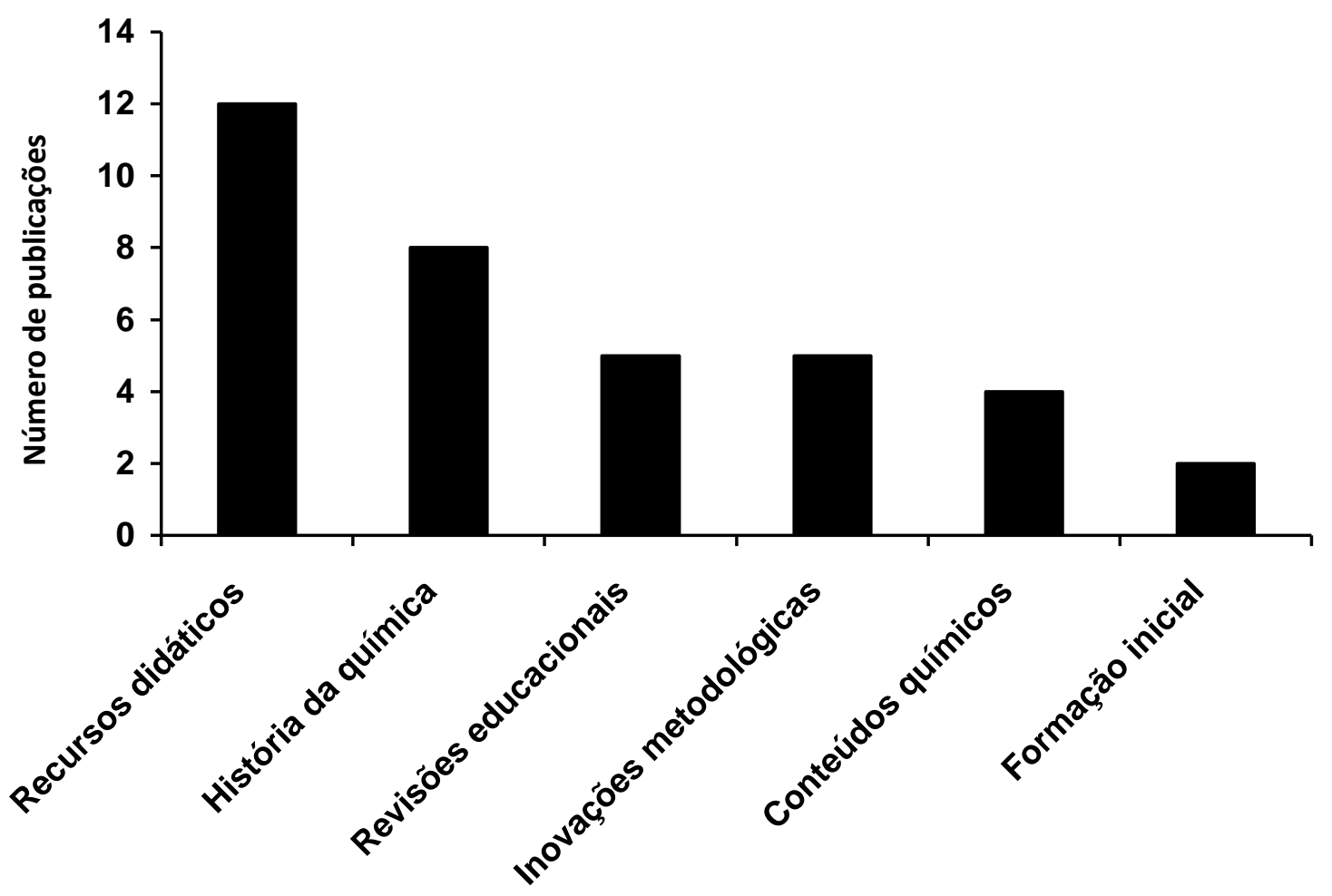

Figura 3. Temática trabalhada no Ensino de Química de 2000 a 2014.

As observações referentes a Figura 2 permitem inferir que há uma preocupação na busca de recursos que permitam a superação no fenômeno da repetência e evasão escolar, e que o ensino possa ser um momento privilegiado de exercitar a imaginação e com isso ser uma fonte de prazer, principalmente quando esses materiais didáticos tem um apelo lúdico que contemple a motivação, gerada pelo desafio, acarretando o desenvolvimento de estratégias de resolução de problemas, a avaliação das decisões tomadas e a familiarização com termos e conceitos apresentados (CARVALHO, et al., 2012; SOUZA; SILVA, 2012).

Bem como, há indicativos sobre uma perspectiva de trabalho com temáticas que levam preocupação com a abordagem sobre os impactos das atividades científicas e tecnológicas na sociedade moderna que implica em uma alfabetização científica da população de forma que ela possa exercer sua cidadania de forma ativa e crítica na tomada de decisões, não deixando isso só a cargo dos especialistas (CACHAPUZ et al, 2011; SCHNETZLER, 2004). 


\section{CONCLUSÕES}

A área de Pesquisa em Ensino de Química tem se desenvolvido e amadurecido no Brasil com trabalhos abordando temas atuais publicados em periódicos especializados e disponibilizados a toda comunidade. Acesso totalmente livre.

O estudo aponta maior preocupação com o Ensino superior, nos quais o currículo de Química é ensinado com mais objetividade, seguido da educação do ensino médio, onde são identificados mais problemas conceituais e carência de recursos didáticos, visto uma parte considerável das escolas públicas não disporem de laboratório onde os jovens possam experenciar o que é visto teoricamente. Observa-se a falta de interesse no ensino fundamental e na formação inicial que é a base para o conhecimento das demais fases.

Estes resultados demonstram a urgente necessidade de uma maior atenção para as séries iniciais e o investimento de pesquisas para outras regiões do país e do estado do Piauí, especificamente.

\section{REFERÊNCIAS}

BRANCO, F. S. C. et al. Ressonância magnética nuclear de substâncias organofluoradas: um desafio no ensino de espectroscopia. Química Nova, Vol. 38, No. 9, 1237-1246, 2015.

CACHAPUZ, A. et al. A Emergência da Didáctica das Ciências como Campo Específico de Conhecimento. Revista Portuguesa de Educação, v.14, n.1, p.155-195, 2001.

CACHAPUZ, A. et al. A necessária renovação do ensino das ciências. 2 ed. São Paulo: Cortez, 2011.

CARvalho, A. M. P., et al. Ensino de ciências: unindo a pesquisa e a prática. São Paulo: Cengage Learning, 2012.

FERREIRA, N. S. A. As pesquisas denominadas "estado da arte". Educação e Sociedade, 23(79): 257-272. 2002.

FRANCISCO, C. A. QUEIROZ, S. L. Novas tecnologias no ensino de Química: um olhar sobre teses e dissertações brasileiras. $33{ }^{a}$ Reunião Anual da Sociedade Brasileira de Química. Disponível em: http://sec.sbq.org.br/cdrom/33ra/resumos/T0412-1.pdf. Acesso em: 29 mar. 2016. 
MARCONDES, M. E. R. Proposições metodológicas para o ensino de química: oficinas temáticas para a aprendizagem da ciência e o desenvolvimento da cidadania. Em extensão, Uberlândia, V. 7, 2008.

MIRANDA, M. S.; SUAR, R. C.; MARCONDES, M. E. R. Promovendo a alfabetização científica por meio de ensino investigativo no ensino médio de química: contribuições para a formação inicial docente. Revista Ensaio, v.17, n. 3, p. 555-583, 2015.

NUNES, A. O. et al. Experimentação pedagógica - relações ctsa na formação inicial do licenciando em química. Enseñanza de las Ciencias, Número Extra VIII Congreso Internacional sobre Investigación en Didáctica de las Ciencias, Barcelona, p. 1987-1991, 2009.

SILVA, L. P.; MARCONDES, M. E. R. Materiais didáticos elaborados por professores de química na perspectiva CTS: uma análise das unidades produzidas e das reflexões dos autores. Ciência e Educação, v. 21, n. 1, p. 65-83, 2015.

SANTOS Jr. J. B. et al. Um estudo comparativo entre a atividade experimental e a simulação por computador na aprendizagem de eletroquímica. Revista Electrónica de Enseñanza de las Ciencias v. 15, $\mathrm{n}^{\mathrm{o}}$ 2, p. 312-330, 2016.

SOUZA, H. Y. S.; SILVA, C. K. O. Dados orgânicos: um jogo didático no ensino de química. HOLOS, v. 3, p. 107-121, 2012.

RAMOS, R. M.; MUÑOZ, L. A. La enseñanza de la química ambiental: Una propuesta fundamentada en la controversia científica y la resolución de problemasLa enseñanza de la química ambiental: Una propuesta fundamentada en la controversia científica y la resolución de problemas. Revista de la Facultad de Ciencia y Tecnología - Tecné, Episteme y Didaxis, v. 38, p. 133-146, 2015.

ROGADO, J. R. A dimensão histórica da ciência presente nas investigações em educação química no brasil (1997-2005). Enseñanza de las Ciencias, Número Extra VIII Congreso Internacional sobre Investigación en Didáctica de las Ciencias, Barcelona, p. 1947-1950, 2009.

SCHNETZLER, R. P. A pesquisa no ensino de Química e a importância da Química Nova na Escola. Química Nova na Escola, nº 20, p. 49-54, 2004.

VALDERRAMA, L. et al. Proposta experimental didática para o ensino de análise de componentes principais. Química Nova, v. 39, nº. 2, p. 245-249, 2016. 